\title{
Identification of expressed genes during compatible interaction between stripe rust (Puccinia striiformis) and wheat using a cDNA
} library

\author{
Jinbiao $\mathrm{Ma}^{1}$, Xueling Huang ${ }^{2}$, Xiaojie Wang ${ }^{2}$, Xianming Chen ${ }^{3}$, Zhipeng $\mathrm{Qu}^{2}$, \\ Lili Huang ${ }^{2}$ and Zhensheng Kang*1,2
}

Address: ${ }^{1}$ College of Life Sciences, Northwest A\&F University, Yangling, Shaanxi, 712100, PR China, ${ }^{2}$ College of Plant Protection and Shaanxi Key Laboratory of Molecular Biology for Agriculture, Northwest A\&F University, Yangling, Shaanxi, 712100, PR China and ${ }^{3}$ USDA-ARS and Department of Plant Pathology, Washington State University, Pullman, WA 99164-6430, USA

Email: Jinbiao Ma - mjb780510@163.com; Xueling Huang - xiaoxiong79221@yahoo.com.cn; Xiaojie Wang - wangxiaojie@nwsuaf.edu.cn; Xianming Chen - xianming@wsu.edu; Zhipeng Qu - quzp2005@126.com; Lili Huang - huanglili@nwsuaf.edu.cn;

Zhensheng Kang* - kangzs@nwsuaf.edu.cn

* Corresponding author

This article is available from: http://www.biomedcentral.com//47/-2/64/10/586

(C) 2009 Ma et al; licensee BioMed Central Ltd.

This is an Open Access article distributed under the terms of the Creative Commons Attribution License (http://creativecommons.org/licenses/by/2.0), which permits unrestricted use, distribution, and reproduction in any medium, provided the original work is properly cited.

\begin{abstract}
Background: Wheat stripe rust, caused by Puccinia striiformis f. sp. tritici (Pst), is one of the most destructive diseases of wheat worldwide. To establish compatibility with the host, Pst forms special infection structures to invade the plant with minimal damage to host cells. Although compatible interaction between wheat and Pst has been studied using various approaches, research on molecular mechanisms of the interaction is limited. The aim of this study was to develop an EST database of wheat infected by Pst in order to determine transcription profiles of genes involved in compatible wheat-Pst interaction.
\end{abstract}

Results: Total RNA, extracted from susceptible infected wheat leaves harvested at 3, 5 and 8 days post inoculation (dpi), was used to create a cDNA library, from which 5,793 ESTs with high quality were obtained and clustered into 583 contigs and 2,160 singletons to give a set of 2,743 unisequences (GenBank accessions: GR302385 to GR305 I27). The BLASTx program was used to search for homologous genes of the unisequences in the GenBank non-redundant protein database. Of the 2,743 unisequences, $52.8 \%$ (the largest category) were highly homologous to plant genes; $16.3 \%$ to fungal genes and $30 \%$ of no-hit. The functional classification of all ESTs was established based on the database entry giving the best E-value using the Bevan's classification categories. About $50 \%$ of the ESTs were significantly homologous to genes encoding proteins with known functions; $20 \%$ were similar to genes encoding proteins with unknown functions and $30 \%$ did not have significant homology to any sequence in the database. The quantitative real-time PCR (qRT-PCR) analysis determined the transcription profiles and their involvement in the wheat-Pst interaction for seven of the gene.

Conclusion: The cDNA library is useful for identifying the functional genes involved in the wheatPst compatible interaction, and established a new database for studying Pst pathogenesis genes and wheat defense genes. The transcription patterns of seven genes were confirmed by the QRT-PCR assay to be differentially expressed in wheat-Pst compatible and incompatible interaction. 


\section{Background}

Rust fungi are obligate biotrophic pathogens responsible for many economically important plant diseases, particularly on cereals. Puccinia striiformis Westend. f.sp. tritici Eriks. (Pst) causes wheat stripe rust, a devastating disease in many wheat growing areas of the world. Yield losses caused by stripe rust over a large area can be up to $50 \%$ [14]. Chemicals have been used to control the disease, but the most cost-effective strategy to reduce the threat of the disease is through growing resistant wheat cultivars. However, cultivars with race-specific resistance genes may become susceptible to the disease when new virulent races of the pathogen emerge. Novel methods to control the disease are needed to be developed, which requires a better understanding of the interactions between the host and pathogen, especially genes of the pathogen expressed in the course of the infection process of the compatible interaction to identify new targets for disease control. Traditionally, incompatible interactions have received much more attention than compatible interaction in order to understand the host resistance mechanism as well as to discover and use resistance genes $[5,6]$. Recently, studies have been reported on pathogen factors that promote compatible interactions and disease development in plant tissue $[7,8]$.

Phenotypically, the compatible and incompatible interactions of wheat-Pst are obviously different. Necrotic spots or stripes can be observed after inoculation in the incompatible interaction and the ceasing development of infection hyphae at an early stage can be observed using a microscope $[1,9,10]$. This phenomenon is regarded as hypersensitive response (HR) and also termed programmed cell death (PCD) $[11,12]$. In an incompatible interaction the host tissue sacrifices the infected cell or several cells around the infection site to prevent further growth of the biotrophic pathogen. In a compatible interaction, the biotrophic fungus Pst [13] is armed with a complex strategy to avoid destructive effects in the host tissue during the processes of infection and spread. When urediniospores land on leaf surfaces of host plants, they germinate under suitable conditions of humidity and temperature, and the germ tubes grow toward stomata. Once germ tubes reach stomatal guard cells, they directly enter into the host leaf tissue through stomata or occasionally form appressoria on stomata [14]. From an appressorium, a penetration peg grows through the stomatal opening and develops into a vesicle in the substomatal cavity. Infection hyphae start growing in the intercellular spaces of the host tissue, and when the tip of an infection hypha reaches the cell wall of its host, a haustorial mother cell is formed. From the haustorial mother cell, a narrow haustorial neck penetrates the wall of the mesophyll cell and develops into a haustorium, which establishes an obligate biotrophic relationship with the living plant cell by redirecting the host's metabolism to meet nutritional needs of the pathogen without causing immediate death of host cells $[15,16]$. The lack of immediate host cell death in plant-pathogen interactions might be achieved by the pathogen through avoiding host recognition or expressing factors suppressing host defense responses. The Pst infection hyphae grow between host cells with haustoria in the pericellular space of host cells. It seems that specific host genes and proteins are targeted by biotrophic parasites to achieve these goals, and as such they may be considered as compatibility factors that are essential for successful pathogenesis.

In the recent years, the expressed sequence tags (ESTs) have been studied in many compatible interactions between rust fungi and their hosts. Numerous genes were reported as virulent or pathogenesis factors that may have distinct functions in the compatible plant-pathogen interaction. Hahn and Mendgen [17] isolated haustoria from Vicia faba leaves infected with Uromyces fabae and constructed a haustorium-specific cDNA library. From the library, they identified 31 in-planta induced genes (PIGs) that were specifically expressed in infected plants, but weakly or not expressed urediniospores. In an effort to interpret the molecular basis of the leaf rust pathogenwheat compatible interaction, the suppression subtractive hybridization and CDNA-AFLP techniques were used $[18,19]$. The ESTs originating from infected wheat leaves were obtained and a proportion of them were likely of fungal origin. The differential expression analysis indicated that most of these genes were specific in rustinfected leaves, and the majority of induced genes showed homology to known PIGs or virulence genes in other fungi. It is predicted that these genes have common functions when the parasites infect their hosts. Recently, an EST library was constructed from fully susceptible wheat leaves inoculated with $P$. graminis f.sp. tritici (the wheat stem rust pathogen) and most ESTs showed similarities to fungal sequences. Quantitative RT-PCR (qRT-PCR) was used to test the expression patterns of fungal ESTs from different organisms including rust-infected leaves [20]. To identify genes expressed at a special physiological stage, a cDNA library of germinating Phakopsora pachyrhizi urediniospores was constructed and $52 \%$ of a total of 908 ESTs were found no significant similarity to the entries in the public protein databases [21]. The result indicates that our knowledge of genes expressed in biotrophic pathogens is still limited.

Puccinia striiformis f. sp. tritici causes severe yield losses in wheat, but the information of gene expressions in infected susceptible wheat cultivars is scanty. To obtain insights into the molecular basis of the compatible interaction between wheat and Pst, we report here an EST library constructed from fully susceptible wheat leaves infected with 
a highly virulent pathotype of Pst. In total, 5,793 ESTs and from which 2,743 unisequences were obtained. The qRTPCR analysis of a number of the ESTs revealed the expression patterns of some important genes involved in the compatible interaction. The results indicate that the EST library is useful for exploring pathogen genes expressed during pathogenesis and plant genes responding to the pathogen in the infection course.

\section{Results and Discussion Construction of a cDNA library from wheat leaves challenged by Pst}

To expand the knowledge of potential genes involved in the wheat-Pst compatible interaction, a cDNA library from the wheat genotype Suwon 11 inoculated with Chinese Pst race CYR31 was constructed. This interaction generated severe disease symptoms (Fig. 1). Three developmental stages of Pst in the infected wheat leaves were selected. 1) From 1 to 3 days post inoculation (dpi). During this early infection stage, Pst sequentially forms the infection peg, infection hyphae, haustorial mother cell and primary haustoria that initiate the pathogen biotrophic stage. From this point, the further life cycle of the pathogen completely depends on the metabolism of the host tissue [1]. 2) The growth of Pst infectious hyphae takes place inside its host and continues from 3 to $7 \mathrm{dpi}$. Based on a study on leaf rust of barley, $81-85 \%$ of the volumes of the fungus were mycelium and 15-19\% haustoria. Visible symptoms of infection become apparent by $7 \mathrm{dpi}$ as macroscopic chlorotic patches [22]. 3) In these chlorotic patches the fungus starts sporulating $8 \mathrm{dpi}$. To representing the important infection stages, infected wheat leaves were harvested at 3,5 and 8 dpi and used for total RNA extraction. $6.3 \times 10^{6}$ pfumL $\mathrm{m}^{-1}$ for primary library titer, 9.7 $\times 10^{9}$ pfumL $^{-1}$ for amplified library titer, and $98 \%$ recom-

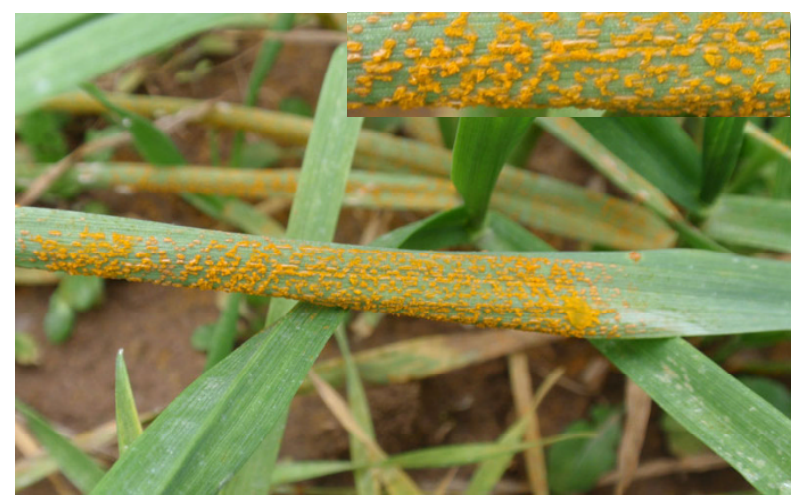

Figure I

A fully susceptible symptom of Puccinia striiformis $f$. sp. tritici-wheat compatible interaction. The I5 dpi wheat leaves from wheat plants Suwon II inoculated with $P$. striiforms f. sp. tritici race CYR3I, which generates a compatible interaction with heavy sporulation from rust uredia. binants were obtained. Inserted cDNA lengths ranged from $0.5 \mathrm{~kb}$ to $2.2 \mathrm{~kb}$.

\section{cDNA sequencing and analyses}

From 6,002 randomly picked clones that were sequenced from 5' end, 5,793 ESTs with high quality were obtained and clustered into 583 contigs and 2,160 singletons to give a set of 2,743 unique sequences (unisequences) (Fig. 2). EST similarity search using the BLASTx program was carried out by comparing sequences in the non-redundant protein database of the GenBank. About 50\% of the ESTs showed significant matches to genes encoding proteins with known functions; $20 \%$ of the ESTs were similar to genes encoding proteins with unknown functions; and $30 \%$ of the ESTs did not have significant similarities to any sequences in the database.

In the library, 52.8\% ESTs, which was the largest category, had the highest homology with plant genes (Additional file 1) $16.3 \%$ ESTs had significant homology with fungal genes (Additional file 2 and $30 \%$ had no hits (Additional file 3). Of the 812 ESTs in the no-hit category, 101 were isolated more than twice. WRIC_47 was isolated 32 times and WRIC_557 18 times. The high frequencies of these genes in the EST library might indicate their involvement in pathogenicity and virulence. In some cases, the lack of similarity to protein database entries could be due to the sequence being derived from the 5 ' non-translated region of the cDNA [23].

\section{Functional annotation and analysis}

To conduct functional classification of the wheat-Pst compatible interaction, each unisequence EST was compared with the non-redundant database from the NCBI using the BLASTX algorithms [24]. The functional classification of all ESTs was established based on the database entry giving the best E-value and the categories defined by Bevan et al. [25]. The unigenes homologous to plant genes were classified into 13 functional categories based on the cellular roles of expressed genes (Fig. 3). The largest category counted for $26 \%$ of the classified ESTs with putative functions as enzymes of energy and metabolism. The second largest was the category of enzymes with unknown and hypothetic protein, counting for $18 \%$ of the classified ESTs. The third category was disease/defense proteins, counting for $13 \%$ of the ESTs.

\section{Genes homologous to those with known functions in other fungal pathogens}

Among the 2,743 unisequences, 446 showed homologies to fungal genes from model fungal species, such as Cryptococcus neoformans, Neurospora crassa, Aspergillus nidulans, Saccharomyces cerevisiae and Candida albicans, and plant pathogenic fungi such as Ustilago maydis, P. triticina, Magnaporthe grisea, Eremothecium gossypii, Uromyces appendicu- 


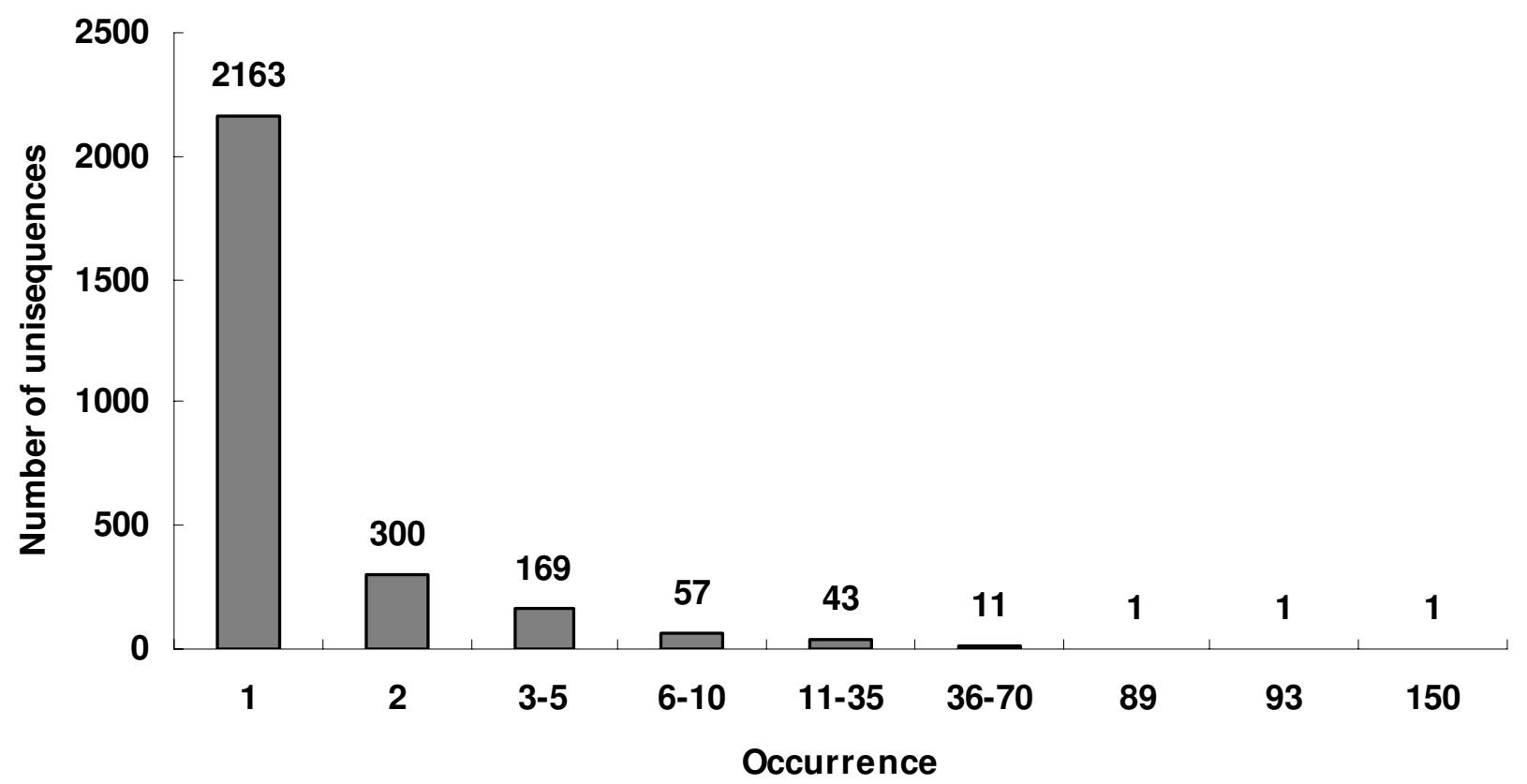

Figure 2

Frequencies of EST clones derived from a Puccinia striiformis f. sp. tritici-wheat compatible interaction. The number of ESTs is shown above the histograph for each number of occurrences. After sequencing 6,002 clones, 5,793 ESTs with high quality were obtained and clustered into 583 contigs and 2, I63 singletons, resulting in 2,746 unique sequences (unisequences).

latus and $P$. graminis. From the fungal ESTs involved in the wheat-Pst compatible interaction, we identified 15 genes homologous to other rust fungal genes (Table 1).

Three genes described as PIGs as defined by Hahn and Mendgen [17] were detected. WRIC_348 was highly homologous to PIG28, which was reported to be continuously up-regulated in infected broad bean leaves from 12or 18-h infection structures and culminate in haustoria and infected leaves infected with $U$. fabae. WRIC_348, which was detected 10 times in the wheat-Pst compatible interaction library, is predicted to encode a protein strongly resembling peptidyl-prolyl cis-trans isomerases (PPIase) and cyclophilin. PPIase is considered to catalyze the isomerization of proline-containing peptide bonds and assists in the folding of proteins [26]. The cytoplasmic PPIase also was induced by heat shock in yeast [27]. Gene encoding cyclophilins were also specifically expressed in $U$. fabae and P. triticina $[17,19]$.

Two ESTs, WRIS_316 and WRIS_1608, were similar to genes THI1 (PIG1) and THI2 (PIG4) expressed in haustoria of U. fabae [28]. THI1 and THI2 were found to be highly expressed in haustoria of $U$. fabae, representing approximately $5 \%$ of the haustorial mRNA and homolo- gous to genes involved in thiamine (vitamin B1) biosynthesis $[17,29]$. This indicates that haustoria not only function in uptaking nutrients from plants but also involved in biosynthesis of fungal metabolites $[28,30]$, an additional role for haustoria in the biosynthesis of fundamental metabolites that might not be provided in sufficient amounts by the host [28]. The expression patterns of THI1 and THI2 were very similar. Their transcription levels increased in germinated urediniospores in vitro, and were also accumulated to very high levels in haustoria and rust-infected host leaves. No expression was observed in healthy plants, urediniospores, and early infection structures of the pathogens. Also the isolation of such genes from the wheat leaves infected by $P$. triticina has been reported [19]. Our results together with the previous reports indicate that the functions of these genes are essential in obligate biotrophic rust fungi.

WRIS_2031 and WRIS_3511 were predicted to encode chitinase because of their best homology to the fungal chitinase gene of $P$. triticina. Both genes are up-regulated from 5 dpi $[17,18]$. Since chitin represents a main component of the cell wall of Pst, it may be assumed that chitinases play an essential role in cell wall synthesis in the tips of intercellularly spreading hyphae. 


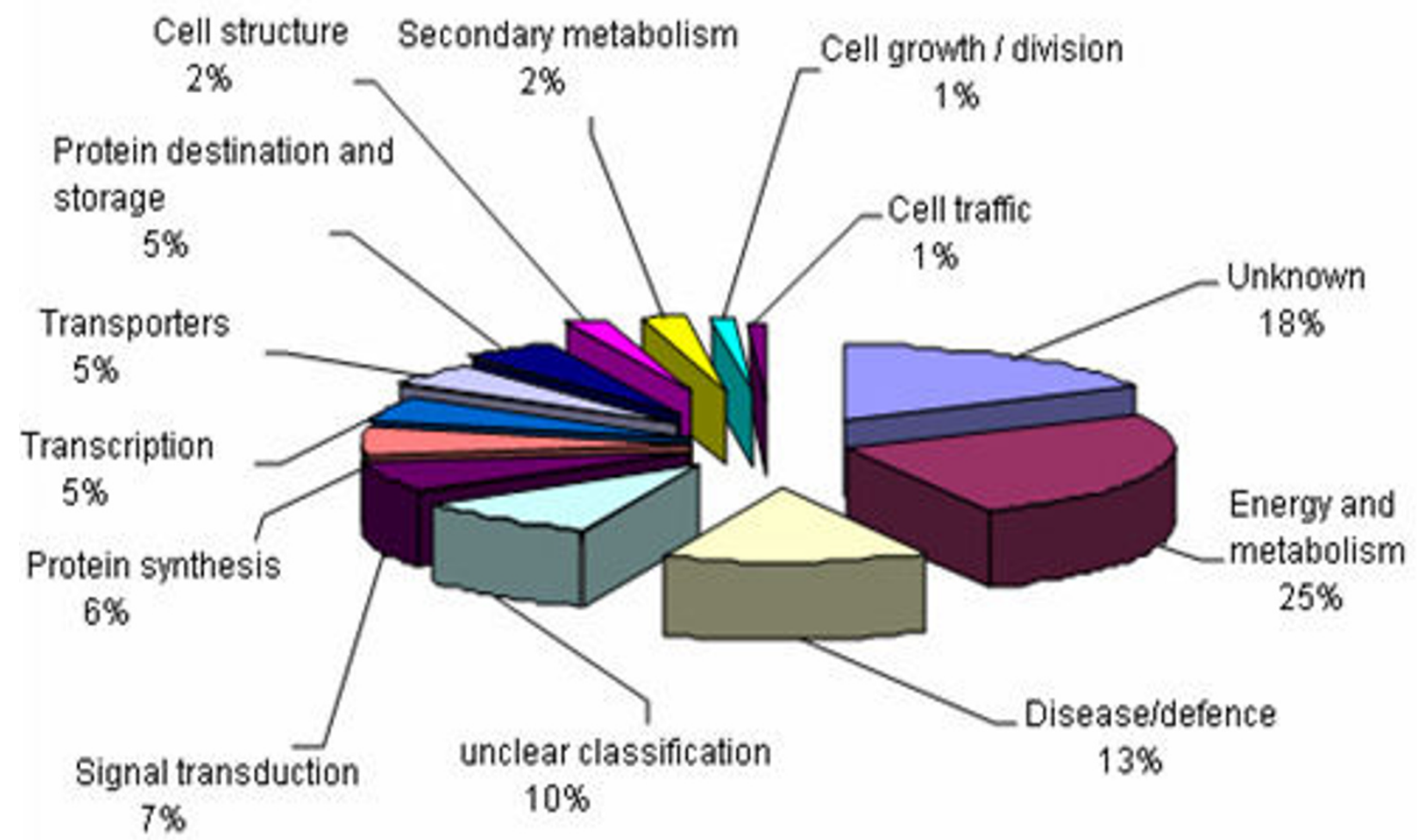

Figure 3

The functional classification of ESTs from Puccinia striiformis f. sp. tritici-wheat compatible interaction with significant similarities to plant genes in the GenBank database. The homologies of unigenes to plant genes were classified into I 3 functional categories based on their cellular roles. About $26 \%$ of the ESTs showed significant homology to enzymes involved in energy and metabolism, representing the largest category. 18\% ESTs showed homologies to genes of unknown function and hypothetic proteins in the Genbank database.

WRIC_312 was assembled with two ESTs and predicted to be a rust transferred protein (RTP) because of its similarity to genes in Uromyces striatus. RTP was originally described as PIG7 among the 31 PIGs isolated from the broad bean leaves infected by U. fabae [17]. An immuno-fluorescence staining test provided evidence that RTP protein represents a secreted protein which is transferred from the rust haustoria into the nucleus of the infected cells of $V$. faba. This was the first report demonstrating that a fungal protein was transferred to the nucleus of its host. It has been suggested that the RTP protein plays an important role in the biotrophic interaction between the parasite and its host [31]. This finding is similar to the bacterial type III secretion system which is well-known for the transfer of virulence proteins into its host cells [32].

The sequence of WRIS_1741 is similar to the gene encoding a cyclase-associated protein 1 (CAP1) from Cryptococcus gattii. CAP1 has three domains. The N-terminal domain binds to adenylyl cyclase and is involved in Ras- responsiveness [33]. The C-terminal domain binds to Gactin and strongly inhibits actin polymerization [34]. The function of the internal, proline-rich domain of CAP is still unclear. Over-expression of an Arabidopsis CAP homologue (AtCAP1) in transgenic tobacco plants reduced expansion of epidermal and mesophyll cells in leaf tissues [35,36]. A CAP1 gene of Candida albicans was identified as a virulence factor when it interacts with its host, and was required for bud-hypha transitions, filamentous growth, and regulation of cAMP levels. C. albicans strains with the CAP1 gene were virulent when they infect their mice host, but the cap1 mutant was avirulent [37].

WRIC_406 was homologous to the chitin deacetylase, which catalyzes deacetylation of chitin to yield chitosan. The chitin deacetylase was specifically regulated in infection structures of $U$. viciae-fabae and seems to help to counter hyphal degradation by plant chitinases [38]. More importantly, chitosan is present in hyphal cell walls 
Table I: Puccinia striiformis f.sp. tritici ESTs with significant homologies to fungal pathogenicity/virulence factors

\begin{tabular}{|c|c|c|c|c|c|}
\hline User ID & Accession No. & Copies & Length & Putative function and Species & E-value \\
\hline WRIC_3I2 & GR302696 & 2 & 951 & rust transferred protein (Uromyces striatus) & $6.00 \mathrm{E}-27$ \\
\hline WRIC_322 & GR302706 & 9 & 978 & differentiation-related protein Infp (Uromyces appendiculatus) & I.00E-14 \\
\hline WRIC_348 & GR302732 & 10 & 785 & PIG28 (Uromyces fabae) & $2.00 \mathrm{E}-53$ \\
\hline WRIC_365 & GR302749 & 2 & 633 & alcohol dehydrogenase (Puccinia triticina) & $6.00 \mathrm{E}-70$ \\
\hline WRIC_406 & GR302790 & 2 & 750 & chitin deacetylase (Flammulina velutipes) & I.00E-III \\
\hline WRIC_495 & GR304563 & 7 & 1333 & elongation factor (Puccinia graminis) & $0.00 \mathrm{E}+00$ \\
\hline WRIS_I74I & GR30326I & I & 666 & CAPI (Cryptococcus gattii) & $1.00 \mathrm{E}-36$ \\
\hline WRIS_I608 & GR3032II & I & 604 & THI2p (Uromyces viciae-fabae) & 8.00E-75 \\
\hline WRIS_I773 & GR303275 & I & 739 & actin (Puccinia graminis) & I.00E-II4 \\
\hline WRIS_45I7 & GR304357 & I & 347 & ATP-binding cassette transporter $\mathrm{ABC} 2$ (Venturia inaequalis) & $2.00 \mathrm{E}-30$ \\
\hline WRIS_I78I & GR303282 & I & 643 & actin (Puccinia graminis) & I.00E-5। \\
\hline WRIS_203I & GR303386 & I & 751 & chitinase (Puccinia triticina) & $2.00 \mathrm{E}-25$ \\
\hline WRIS_316 & GR303865 & I & 619 & PIGI (Uromyces fabae) & $0.00 \mathrm{E}+00$ \\
\hline WRIS_3246 & GR303896 & I & 695 & hypothetical class II chitin synthase (Puccinia graminis) & $9.00 \mathrm{E}-29$ \\
\hline WRIS_35II & GR304003 & I & 790 & chitinase (Puccinia triticina) & $4.00 \mathrm{E}-19$ \\
\hline WRIS_4318 & GR304280 & I & 524 & HSSI (Puccinia graminis) & $0.00 \mathrm{E}+00$ \\
\hline WRIS_574 & GR304877 & I & 574 & alcohol dehydrogenase (Puccinia triticina) & $7.00 \mathrm{E}-82$ \\
\hline WRIS_4003 & GR304I67 & I & 699 & Ras2 (C. neoformans) & I.00E-74 \\
\hline WRIS_36I7 & GR304047 & I & 597 & $A B C$ transporter (Candida albicans) & $2.00 \mathrm{E}-27$ \\
\hline
\end{tabular}

of Pst immediately at the beginning of the plant-pathogen interaction [39]. This indicates that the gene of chitin deacetylase is transcribed specifically at the intimate contact stage of the pathogen with its host tissue. Thus, deacetylation of chitin may protect hyphal cell walls or other infection structures from degrading by plant chitinases [40].

ATP-binding cassette (ABC) transporters are membrane proteins which drive the transport of compounds over the biological membranes. WRIS_3617 and WRIS_4517 are two genes homologous to $\mathrm{ABC}$ transporters of Candida albicans and Venturia inaequalis, respectively. ABC transporters are known to be involved in drug resistance which has been described in filamentous fungi [41]. In plant pathogenic fungi $A B C$ transporters may act as virulence factors which mediate secretion of pathogenicity factors or protecting the fungi against defense compounds produced by host plants $[42,43]$. Thus, ABC transporters may play a dual role in pathogenic fungi resulting in enhancing their virulence. These results strongly suggest that Pst requires the up-regulation of specific $A B C$ transporters for pathogenesis and protection against plant defense mechanisms. Recently, the cloned $\operatorname{Lr} 34 / \mathrm{Yr} 18$ for durable resistance to leaf rust and stripe rust in wheat was found to be a putative ABC transporter [44]. Thus, WRIS_3617 and WRIS_4517 are also likely wheat genes involved in the basal defense during the compatible interaction.

In our cDNA library, WRIS_1157 had a similar sequence to that of a Cryptococcus neoformans transketolase gene, which is an enzyme involved in primary carbohydrate metabolism. The finding of WRIS_1157 may provide evi- dence for the normal biosynthetic capabilities of rust fungi when it intrudes into the plant tissue as previously hypothesized [17].

Actin is an important framework compound and ubiquitous in various organisms. We identified the high occurrence of WRIS_1773 and WRIS_1781, which were predicted to encode actin proteins based on their high sequence similarities (E-value were 1e-144 and 1e-51, respectively) to those of $P$. graminis. This provided evidence that both Pst and P. graminis have developed common structures in susceptible wheat cultivars.

\section{Quantitative real-time PCR (qRT-PCR) analysis of candidate pathogenicity-related factors}

Seven genes were selected to conduct the GRT-PCR analysis for transcription levels at different developmental and infection stages (Table 2). Four of these genes were found from wheat and three from Pst based on the results of the BLAST search. The four wheat genes, WRIS_671 (BAX inhibitor 1), WRIS_233 (cyclophilin), WRIS_1264 (defender against cell death 2) and WRIC_116 (putative cell death suppressor) were predicted to be related to defense against cell death at different infection stages of wheat-Pst interaction based on the expression patterns (Fig. 4). Bax inhibitor 1 (BI-1) was reported as the cell death suppressor of Bax-induced cell death in mammals and plants $[45,46]$. The expression of BI-1 gene was rapidly up-regulated during abiotic and biotic stresses [47]. The over-expression and knock-down of the BI-1 in Arabidopsis plants did not affect the plant growth under general growth conditions [48]. These results demonstrate that BI1 is specifically regulated by some environmental factors. 
In the compatible and incompatible interactions of wheat and Pst, BI-1 had a similar transcription expression pattern. It was up-regulated at $24 \mathrm{hpi}$ after inoculation with nearly equal transcription levels and then slightly decreased at $48 \mathrm{hpi}$ and reached the lowest levels by 72 hpi in the compatible interaction, but decreased drastically at 48 hpi in the incompatible interaction. However, it was again up-regulated abruptly $72 \mathrm{hpi}$ in the incompatible interaction.

In the compatible interaction, WRIS_233 (cyclophilin) was up-regulated slightly at $24 \mathrm{hpi}$, but subsequently down-regulated, while in the incompatible interaction, the gene was drastically up-regulated at 24 and $120 \mathrm{hpi}$. These results suggest that cyclophilin has more functional activities in the incompatible interaction. In contrast, in the compatible interaction, cyclophilin was down-regulated at 24 hpi. WRIS_1264 is a defender against cell death 2 (DAD2), which is different from DAD1 at seven amino acid residues. DAD1 is a negative regulator of programmed cell death (PCD) identified as a part of the oligo saccharyltransferase (OST) complex. It has been reported also as a cell death suppressor protein, whose defect at restrictive temperature causes PCD in a hamster mutant cell line [49]. PCD results in a hypersensitive response of plants in which cells invaded by the pathogen and surrounding cells die in order to limit pathogen spread $[50,51]$. PCD is one of the vital characters of incompatible plant-pathogen interactions. DAD2 was highly expressed in the wheat-Pst interaction as two peaks during the early infection stage at 24 and $120 \mathrm{hpi}$. In the compatible interaction, it dropped to the lowest level at $72 \mathrm{hpi}$. It is unclear whether DAD modulates the process of PCD in the wheat-Pst compatible interaction. However, DAD2 expression at high levels at the infection stages indicates that it plays likely a crucial but so far an unknown role. Another gene (WRIC_116) was labeled as putative cell death suppressor, which doesn't have a clear function annotation. It was up-regulated at 24 and $48 \mathrm{hpi}$ at high levels and down-regulated at 72 and $120 \mathrm{hpi}$ in the compatible interaction, but up-regulated only at $48 \mathrm{hpi}$ in the incompatible interaction. Cell death suppressor was an anti-apoptosis protein in transgenic plants and was demonstrated as Bcl-2, Bcl-XL and Ced-9 to provide protection from pathogens [52,53]. As a biotrophic pathogen, Pst intrudes into the plant tissue by avoiding damage to plant tissue in the compatible interaction, a voluntary cell death can restrict establishing infection and spreading of the pathogen.

The expression patterns of the three Pst genes are shown in Fig. 5. The sequences of WRIS_316 and WRIC_1608 were similar to the pathogenesis related proteins PIG1 (THI1p) and PIG4 (THI2p) from U. fabae, respectively. Two ESTs were homologous to genes involved in thia-
Table 2: Sequences of primers used for qRT-PCR

\begin{tabular}{ll}
\hline Unigenes ID & Primer sequences(5'--3') \\
\hline WRIS_67I & $\begin{array}{l}\text { Forward GGCTACGACTCCCTCAAGAAC } \\
\text { Reverse TAGACTGGCACCGAGAACATC }\end{array}$ \\
\hline WRIS_233 & $\begin{array}{l}\text { Forward GGGAGAGATACCAACGGAT } \\
\text { Reverse CGCTTCTACAACGGAAACTA }\end{array}$ \\
\hline WRIS_1264 & $\begin{array}{l}\text { Forward ACGCCCACGAATCTCAAG } \\
\text { Reverse AGGCAAACACCAAGCACTG }\end{array}$ \\
\hline WRIC_I16 & $\begin{array}{l}\text { Forward CAGCCTGACTGGTATGGAAG } \\
\text { Reverse ATGCTTCTGGCGGTCGTAG }\end{array}$ \\
\hline WRIC_316 & $\begin{array}{l}\text { Forward TCTCTGTCCTTCTCAACTGGC } \\
\text { Reverse TGGATCATAGCTTTGCATCC }\end{array}$ \\
\hline WRIC_1608 & $\begin{array}{l}\text { Forward TCGCACCCATCAAGGAGCAT } \\
\text { Reverse GCCACCCAACCAACAACCAC }\end{array}$ \\
\hline Wheat I8s RNA & $\begin{array}{l}\text { Forward CTGAGAAACGGCTACCACAT } \\
\text { Reverse CCCAAGGTCCAACTACGAG }\end{array}$ \\
\hline Reverse CCAGTTATTGCCGTTCACA \\
Reverse GGAGGCATACAAGGAGAGC
\end{tabular}

mine (vitamin B1) biosynthesis in yeast and have a very similar transcriptional pattern. These genes were reported to be mainly transcribed in haustoria and rust-infected broad bean leaves, but were not detected in urediniospores and in infection structures differentiating in vitro until 18 hpi $[17,28]$. PIG1 and PIG4 primers were designed according to the EST sequences generated from the cDNA library from wheat-Pst compatible interaction and the mRNA expression pattern of these two ESTs tested by qRT-PCR. Both genes were remarkably up-regulated at the later stage of Pst development, but no transcripts were detected in the early stage post inoculation and in urediniospores. According to the results of a previous study [54] and our findings, PIG1 and PIG4 have similar transcription patterns in the compatible rust-plant interaction and their expression begin at the stage of haustorial formation.

WRIC_406 is homologue to a chitin deacetylase gene from Flammulina velutipes. The enzyme plays a role in the formation of a protection compound against fungal infection s [55]. In the wheat-Pst compatible interaction, it was highly transcribed at the later stage of Pst development in wheat tissue, but no transcripts were detected in healthy plants, early stages after inoculation and in urediniospores (Fig. 5). According to the character of obligate 

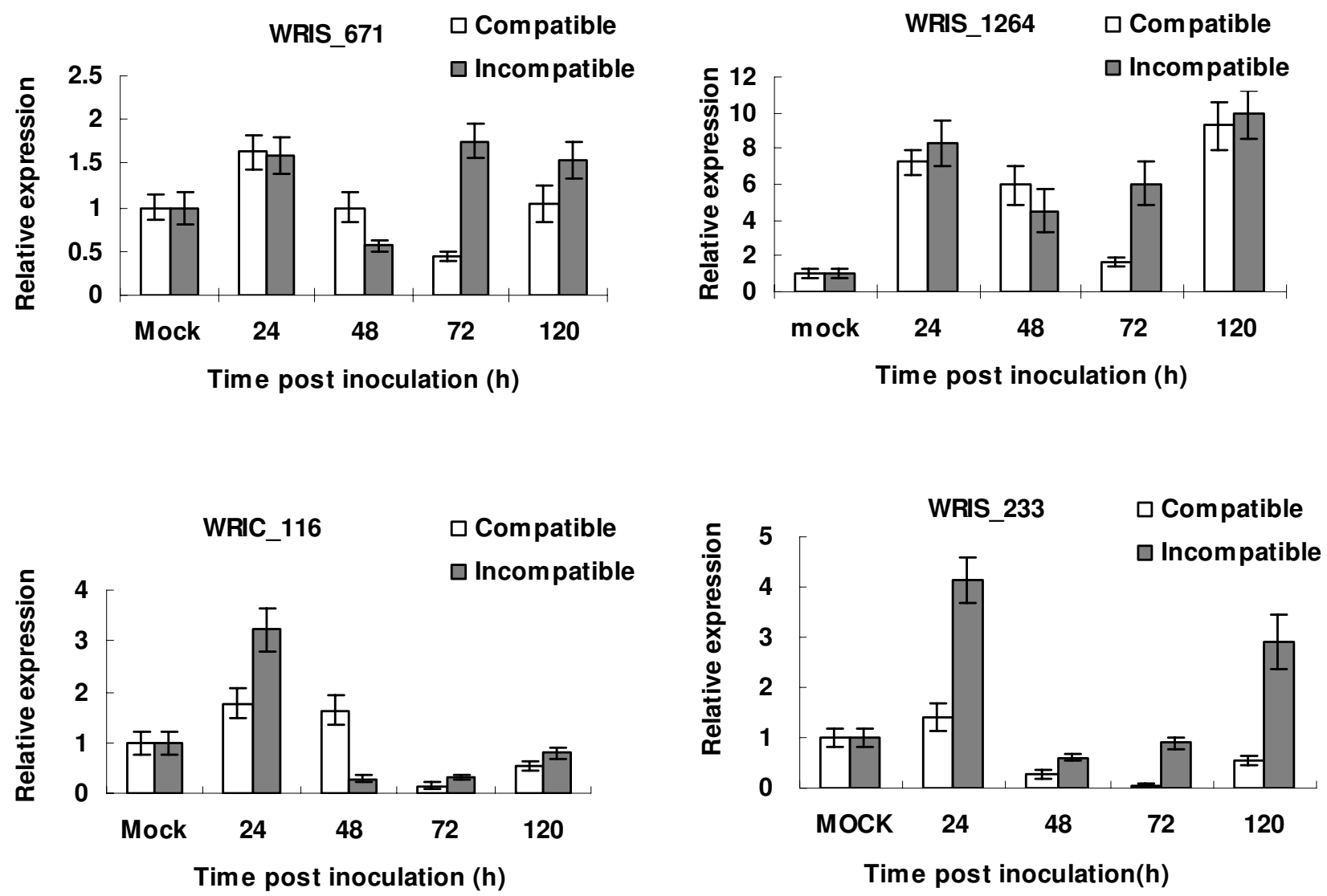

Figure 4

Quantitative RT-PCR assay for the transcription levels of four cell death suppressor-related proteins in the compatible and incompatible interactions of wheat- $\boldsymbol{P}$. striiformis $\mathbf{f}$. sp. tritici. RNA derived from wheat-P. striiformis $f$. sp. tritici. compatible (wheat cultivar Suwon II inoculated with CYR3 I) and incompatible (wheat cultivar Suwon II inoculated with CYR23) interactions was extracted from infected wheat leaves harvested at 0, 24, 48, 72 and I 20 hpi. The $Y$ ax shows relative expressions as folds of the mean expression level of the mock treatment. The mock values for compatible and incompatible interactions are mean values for all time-points as there was no significant difference among the different time points.

biotrophic fungi [16], the reason might be that in the initial phase rust infection structures are not in close contact to the plant cells and expression is not triggered. When more infection hyphae and the rust haustoria are formed, a tight contact to plant cells is established and the real biotrophic phases operate. The host secrets higher activities of chitinases to degrade the chitin in fungal cell walls, however, synthesis of fungal chitin deacetylase is elicited to evade the action of host defense [38].

\section{Conclusion}

From a total of 5,793 ESTs of high quality, 2,743 unisequences were obtained from a cDNA library of a compatible wheat-Pst interaction. The library is useful for identifying the functional genes involved in the wheat-Pst compatible interaction, and has established a new database for studying Pst pathogenesis genes and wheat defense genes. About $50 \%$ of the ESTs were significantly homologous to genes encoding proteins with known functions; $20 \%$ were similar to genes encoding proteins with unknown functions; and 30\% did not have significant homology to any sequence in the database. $52.8 \%$ of the ESTs had the highest homology with plant genes and $16.3 \%$ had significant homology to fungal genes. The transcription patterns of seven genes were determined by qRT-PCR assays, revealing their differential expression patterns in wheat-Pst compatible and incompatible interaction.

\section{Methods}

\section{Plant growth and inoculation}

Chinese Pst races CYR31 and CRY23 used in this study were from the $P$. striiformis $\mathrm{f}$. sp. tritici collection of the College of Plant Protection at Northwest A\&F University. 


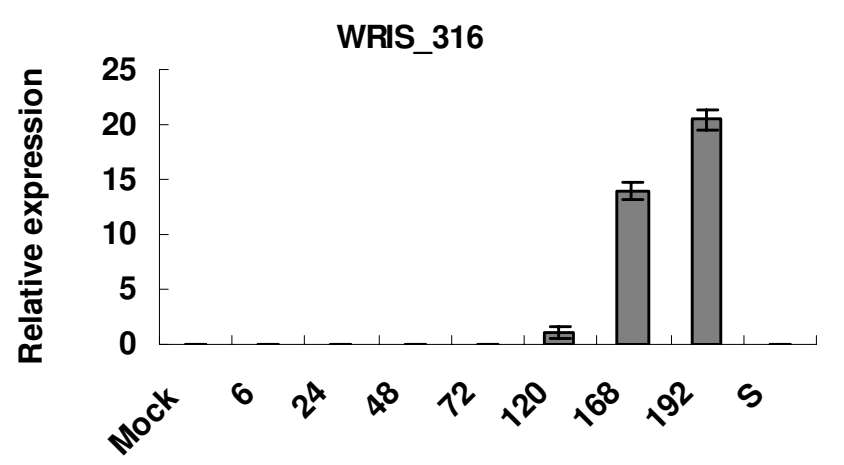

Time post inoculation (h)

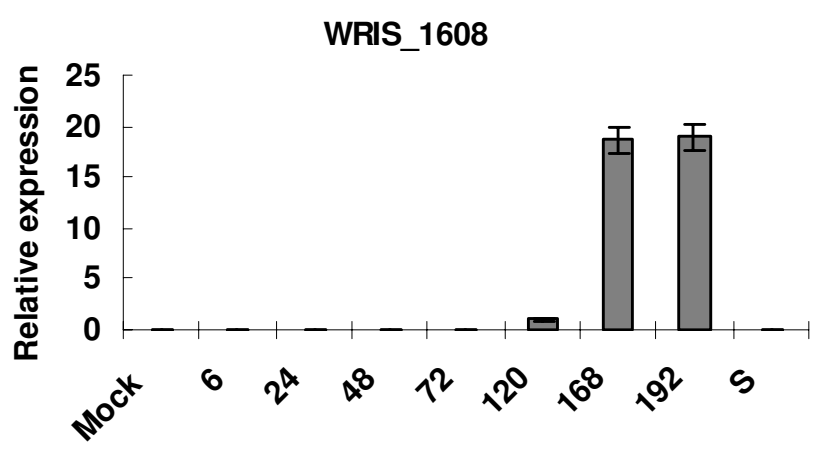

Time post inoculation (h)

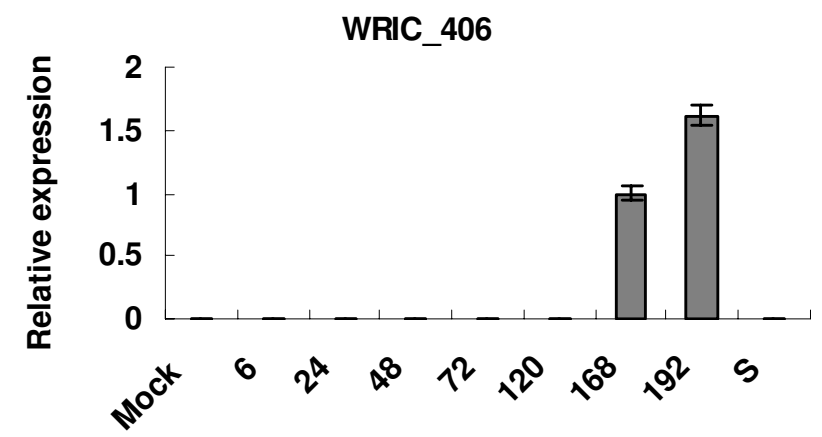

Time post inoculation (h)

\begin{abstract}
Figure 5
Quantitative RT-PCR assay for the transcription levels of three ESTs with significant homologies to fungal genes in the compatible interactions of wheat- Puccinia striiformis f. sp. tritici and urediniospores. RNA derived from compatible (wheat cultivar Suwon II inoculated with CYR3I) interaction was extracted from infected wheat leaves harvested at $0,6,24,48,72,120,168,192$ hpi and urediniospores. The $Y$ ax of the figure shows relative expressions of three fungal homologues genes compared with the mean expression level of the mock treatment. The mock values for compatible interactions are mean values for all time-points as there is no significant difference among the different time points. S, urediniospores.
\end{abstract}

Wheat genotype Suwon 11 was grown in the growth chambers at $15-20^{\circ} \mathrm{C}$. When primary leaves developed, wheat plants were inoculated separately with urediniospores of the pathotypes CYR31 and CYR23. CYR31 produces a compatible interaction and CRY 23 produces an incompatible interaction on Suwon 11. The former combination was used for constructing the compatible interaction cDNA library and qRT-PCR experiments while the later combination was used only in the qRT-PCR analysis for comparison of transcriptional levels of selected genes. Post-inoculated and mock-inoculated plants were kept at high humidity in the dark for $24 \mathrm{~h}$ followed by the regular day-night rhythm in the growth chamber. Primary leaves were harvested at various days post-inoculation (dpi) and immediately frozen in liquid nitrogen.

\section{RNA extraction and CDNA library construction}

Total RNA was extracted from sampled Suwon 11-CYR31 wheat leaves harvested at 3, 5 and 8 dpi using the RNeasy plant Mini kit from QIAGEN. The construction of the cDNA library was carried out using the Clontech SMART cDNA Library Construction Kit. Equal amounts of the total RNA samples from three samples were pooled as the initiating template of ss-cDNA synthesis, ds-cDNA was amplified using LD PCR and digested ds-cDNA was ligated into $\lambda$ TriplEx2 vector and packaged with MaxPlaxTM Lambda Packaging Extract from EPICENTRE according to the manufacturer's instructions. About 10,000 individual phage colonies were transferred to 0.5 $\mathrm{mL}$ micro-centrifuge tubes, containing $1 \times$ Lambda dilu- 
tion buffer supplement with 7 Dimethyl Sulphoxide (DMSO).

\section{cDNA sequencing and analysis}

More than 6,000 clones were randomly selected, and the size of each insert was assessed by PCR amplification with the sequencing primers generated from the two ends of vector and subjected to electrophoresis using 1.5\% agarose gels. Plasmid DNAs were prepared for sequencing reactions using the H.Q. \& Q. plasmid mini kit. Purified plasmid DNA was sequenced from the $5^{\prime}$ end using the Applied Biosystems (ABI) PRISM bigdye terminator kit (Perkin-Elmer) and the ABI Applied Biosystems $3700 x l$ DNA analyzer at the Biotechnology Center of Northwest A\&F University (Yangling, China).

The Phred program was used to trim vector sequences and low-quality sequences from the raw sequencing data, then the processed sequences were clustered into contigs and singletons using the CAP3 software. Sequence similarity search for each unisequence was done using the BLASTX algorithm [25] to identify putative gene homologues in the non-redundant (nr) protein sequence database of the National Center for Biotechnology Information (NCBI).

\section{Real-time PCR}

RNA from compatible (Suwon 11-CYR31) and incompatible (Suwon 11-CYR23) interactions was extracted from infected wheat leaves harvested at $0,24,48,72$ and 120 hpi for four plant homologue genes and 0, 6, 24, 48, 72, 120, 168, $192 \mathrm{hpi}$ and urediniospores for Pst genes. The cDNA was synthesized from $2 \mu \mathrm{g}$ total RNA with $500 \mathrm{ng}$ of 18mer oligo-dT primers and M-MLV reverse transcriptase (Promega). Quantitative RT-PCR was performed on the iQ5 Real-Time PCR Detection System instrument (Bio-Rad), and the primers of quantitative PR-PCR were designed from the differentially expressed ESTs using the Primer 5.0 program. A $18 \mathrm{~s}$ ribosomal RNA sequence from wheat (gi: 15982656) and an actin sequence from $P$. graminis (gi: 1703158) were selected as an endogenous control for plant genes and fungal genes, respectively, to normalize the input RNA and efficiency of reverse transcription between the samples. For quantitative RT-PCR, 3 $\mu \mathrm{L}$ of each template was used in each reaction with 0.12 $\mu \mathrm{M}$ of each primer using iQ SYBR Green Supermix (BioRad). The following cycling program was used: $95^{\circ} \mathrm{C}$ for $60 \mathrm{sec}$, followed by 40 cycles $\left(10 \mathrm{sec}\right.$ at $95^{\circ} \mathrm{C}, 30 \mathrm{sec}$ at $55^{\circ} \mathrm{C}$ and $30 \mathrm{sec}$ at $72^{\circ} \mathrm{C}$ ). All products were subjected to the melting curve analysis between $55^{\circ} \mathrm{C}$ and $95^{\circ} \mathrm{C}$ to determine the specificity of the PCR reaction. Experiments included a non-template control. Quantification of the target gene was assessed using relative standard curves. The plasmid with the target gene was used to prepare standard curves that contained five serial dilutions $\left(10^{-1}\right.$,
$10^{-2}, 10^{-3}, 10^{-4}$ and $\left.10^{-5}\right)$ and gene quantity was determined by the standard curve. The correlation coefficients for the analysis of dilution curve were above 0.99.

Quantitative RT-PCR experiments were done with three biological replicates, the transcription level of target genes were expressed as endogenous control transcripts and the gene expression level in inoculated sample was compared to the average expression level of mock-inoculated sample from all time-points.

\section{Authors' contributions}

JBM: designed experiments, analyzed data and wrote manuscript. XLH: conducted qRT-PCR and analyzed the data. XJW and ZPQ participated in EST sequencing and bioinformatics analysis, LLH: coordinated the experiments and data analyses. XMC: provided advices for experiments and revised manuscript. ZSK: conceived the project, designed the experiments and wrote manuscript. All authors read and approved the final manuscript.

\section{Additional material}

\section{Additional file 1 \\ Unisequences from the compatible interaction between wheat and Puccinia striiformis $f$. $s p$. tritici showing significant similarities to plant genes in the GenBank database. These data provided represent the original EST number and best hit. \\ Click here for file \\ [http://www.biomedcentral.com/content/supplementary/1471- 2164-10-586-S1.DOC]}

\section{Additional file 2}

Unisequences from the compatible interaction between wheat and Puccinia striiformis $f$. sp. tritici showing significant similarities to fungi genes in the GenBank database. These data provide the original EST number and best hit.

Click here for file

[http://www.biomedcentral.com/content/supplementary/14712164-10-586-S2.DOC]

\section{Additional file 3}

Unisequences that do not have any hits in the GenBank databases. These data provides the ESTs that do not have hits in the GenBank databases.

Click here for file

[http://www.biomedcentral.com/content/supplementary/14712164-10-586-S3.DOC]

\section{Acknowledgements}

This study was supported by grants from the National Basic Research Program of China (No. 2006CB 100203); Nature Science Foundation of China (No. 30671350); The Earmarked Fund for Modern Agro-industry Technology Research System of China, and The I I I Project from Ministry of Education of China (B07049). 


\section{References}

I. Li ZQ, Zeng SM: Wheat rust in China (in Chinese) Beijing, China Agriculture Press; 2002.

2. Chen XM: Epidemiology and control of stripe rust [Puccinia striiformis f. sp. tritici] on wheat. Can J Plant Pathol 2005 27:3।4-337.

3. Wan A, Zhao Z, Chen XM, He Z, Jin S, Jia Q, Yao G, Yang J, Wang B, Li G: Wheat stripe rust epidemic and virulence of Puccinia striiformis f. sp. tritici in China in 2002. Plant Dis 2004, 88:896-904.

4. Chen XM, Moore M, Milus EA, Long DL, Line RF, Marshall D, Jackson $\mathrm{L}$ : Wheat stripe rust epidemics and races of Pucciniastriiformis f. sp. tritici in the United States in 2000. Plant Dis 2002, 86:39-46.

5. Chen XM, Line RF: Inheritance of stripe rust resistance inwheat cultivars used to differentiate races of Puccinia striiformis in North America. Phytopathology 1992, 82:633-637.

6. Shi ZX, Chen XM, Line RF, Leung H, Wellings CR: Development of resistance gene analog polymorphism markers for the Yr9 gene resistance to wheat stripe rust. Genome 200I, 44:509-5 I6.

7. Zhang Y, Qu Z, Zheng W, Liu B, Wang X, Xue X, Xu L, Huang L, Han $Q$, Zhao J: Stage-specific gene expression duringurediniospore germination in Puccinia striiformis f. sp tritici. BMC Genomics 2008, 9:203.

8. Ling $P$, Wang $M$, Chen XM, Campbell KG: Construction and characterization of a full-length cDNA library for the wheat stripe rust pathogen (Puccinia striiformis f. sp. tritici). $B M C$ Genomics 2007, 8: 145

9. Kang Z, Huang L, Buchenauer $\mathrm{H}$ : Ultrastructural changes andlocalization of lignin and callose in compatible and incompatible interactions between wheat and Puccinia striiformis. J Plant Dis and Protect 2002, 109:25-37.

10. Kang Z, Wang Y, Huang L, Wei G, Zhao J: Histology andultrastructure of incompatible combination between Puccinia striiformis and wheat cultivars with low reaction type resistance. J Agr Sci Technol China 2003, 2: I I02-III3.

II. Greenberg JT, Yao N: The role and regulation of programmed cell death in plant-pathogen interactions. Cellular Microbiol 2004, 6:20I-2II.

12. Heath MC: Apoptosis, programmed cell death and the hypersensitive response. Eur J Plant Pathol 1998, I 04: I I 7-I 24

13. Mendgen K, Hahn M: Plant infection and the establishment of fungal biotrophy. Trends in Plant Sci 2002, 7:352-356.

14. Zhang H, Han Q, Wang C, Huang L, Zhang Q, Kang Z: Histology and ultra structure of resistant mechanism of a new wheat material Yilipu to Puccinia striiformis (in chinese). Acta Phytopathologica Sinica 2008, 38: I53-I64.

15. Voegele RT, Struck C, Hahn M, Mendgen K: The role of haustoria in sugar supply during infection of broad bean by the rust fungus Uromyces fabae. PNAS 200I, 98:8I33.

16. Panstruga R: Establishing compatibility between plants and obligate biotrophic pathogens. Cur Opinion in Plant Biol 2003 6:320-326

17. Hahn M, Mendgen K: Characterization of in planta-induced rust genes isolated from a haustorium-specific cDNA library. Mol Plant-Microbe Interact 1997, 1 0:427-437.

18. Zhang L, Meakin H, Dickinson M: Isolation of genes expressed during compatible interactions between leaf rust (Puccinia triticina) and wheat using cDNA-AFLP. Mol Plant Pathol 2003, 4:469-477.

19. Thara VK, Fellers JP, Zhou JM: In planta induced genes of Puccinia triticina. Mol Plant Pathol 2003, 4:5I-56.

20. Broeker K, Bernard F, Moerschbacher BM: An EST library from Puccinia graminis f. sp. tritici reveals genes potentially involved in fungal differentiation. FEMS Microbiol Let 2006 , 256:273-28I.

21. Posada-Buitrago ML, Frederick RD: Expressed sequence taganalysis of the soybean rust pathogen Phakopsora pachyrhizi. Fungal Genet Biol 2005, 42:949-962.

22. Kneale J, Farrar JF: The localization and frequency of haustoria in colonies of brown rust on barley leaves. New Phytologist 1985, I 0 I:495-505.

23. Skinner W, Keon J, Hargreaves J: Gene information for fungal plant pathogens from expressed sequences. Cur Opinion in Microbiol 200I, 4:38I-386.
24. Altschul SF, Madden TL, Schaffer AA, Zhang J, Zhang Z, Miller W, Lipman DJ: Gapped BLAST and PSI-BLAST: a new generation of protein database search programs. Nucleic Acids Res 1997, 25:3389.

25. Bevan M, Bancroft I, Bent E, Love K, Goodman H, Dean C, Bergkamp R, Dirkse W, Staveren MV, Stiekema W: Analysis of I.9 Mb of contiguous sequence from chromosome 4 of Arabidopsis thaliana. Nature 1998, 391:485-488.

26. Schonbrunner ER, Schmid FX: Peptidyl-prolyl cis-trans isomerase improves the efficiency of protein disulfide isomerase as a catalyst of protein folding. PNAS 1992, 89:4510-45I3.

27. Sykes K, Gething M, Sambrook J: Proline isomerases function during heat shock. PNAS 1993, 90:5853-5857.

28. Sohn J, Voegele RT, Mendgen K, Hahn M: High level activation of vitamin B I biosynthesis genes in haustoria of the rust fungus Uromyces fabae. Mol Plant-Microbe Interact 2000, I 3:629-636.

29. Jakupovi M, Heintz M, Reichmann P, Mendgen K, Hahn M: Microarray analysis of expressed sequence tags from haustoria of the rust fungus Uromyces fabae. Fungal Genet Biol 2006, 43:8-19.

30. Voegele RT, Mendgen K: Rust haustoria: nutrient uptake and beyond. New Phytologist 2003, I 57:93-100.

31. Kemen E, Kemen AC, Rafiqi M, Hempel U, Mendgen K, Hahn M, Voegele RT: Identification of a protein from rust fungi transferred from haustoria into infected plant cells. Mol PlantMicrobe Interact 2005, I 8: I I30-1 I39.

32. Galán JE, Collmer A: Type III secretion machines: bacterial devices for protein delivery into host cells. Science 1999, 284: 1322.

33. Mintzer KA, Field J: Interactions between adenylylcyclase, CAP and RAS from Saccharomyces cerevisiae. Cellular Signal 1994, 6:68I-694.

34. Freeman NL, Chen Z, Horenstein J, Weber A, Field J: An actin monomer binding activity localizes to the carboxyl-terminal half of the Saccharomyces cerevisiae cyclase-associated protein. Biol Chem 1995, 270:5680-5685.

35. Barrero RA, Umeda M, Yamamura S, Uchimiya H: Over-expression of Arabidopsis CAP causes decreased cell expansion leading to organ size reduction in transgenic tobacco plants. Annals of Botany 2003, 91 :599-603.

36. Deeks MJ, Rodrigues C, Dimmock S, Ketelaar T, Maciver SK, Malho R, Hussey PJ: Arabidopsis CAPI a key regulator of actin organisation and development. / Cell Science 2007, I 20:2609-26 I8.

37. Bahn YS, Sundstrom P: CAPI, an adenylatecyclase-associated protein gene, regulates bud-hypha transitions, filamentous growth, and cyclic AMP levels and is required for virulence of Candida albicans. J Bacteriol 200 I, I 83:32 I I-3223.

38. Deising $\mathrm{H}$, Siegrist $\mathrm{J}$ : Chitin deacetylase activity of the rust Uromyces viciae-fabae is controlled by fungal morphogenesis. FEMS Microbiol Let 1995, I 27:207-2I I.

39. Hadwiger LA, Line RF: Hexosamine accumulations areassociated with the terminated growth of Puccinia striiformis on wheat isolines. Physiol Plant Pathol 1981, I 9:249-255.

40. Siegrist J, Kauss $\mathrm{H}$ : Chitin deacetylase in cucumber leaves infected by Colletotrichum lagenarium. Physiol Mol Plant Pathol 1990, 36:267-275.

41. De Waard MA: Significance of $A B C$ transporters in fungicide sensitivity and resistance. Pestic Sci 1997, 5 I:27 I-275.

42. Del Sorbo G, Schoonbeek H, De Waard MA: Fungal transporters involved in efflux of natural toxic compounds and fungicides. Fungal Genet Biol 2000, 30: I-I5

43. Urban M, Bhargava T, Hamer JE: An ATP-driven efflux pump is a novel pathogenicity factor in rice blast disease. EMBO 1999 , | 8:5 | 2-52|

44. Krattinger SG, Lagudah ES, Spielmeyer W, Singh RP, Huerta-Espino I McFadden H, Bossolini E, Selter LL, Keller B: Putative ABC transporter confers durable resistance to multiple fungal pathogens in wheat. Science 2009, 323:1360-1363.

45. Xu Q, Reed JC: Bax inhibitor-I, a mammalian apoptosis suppressor identified by functional screening in yeast. Molr Cell 1998, I:337-346

46. Kawai-Yamada M, Ohori $\mathrm{Y}$, Uchimiya $\mathrm{H}$ : Dissection of Arabidopsis Bax inhibitor-I suppressing Bax-, hydrogen peroxide-, and salicylic acid-induced cell death. Plant Cell 2004, 1 6:21-32.

47. Sanchez P, de Torres Zabala M, Grant M: AtBI-I, a plant homologue of Bax inhibitor-I, suppresses Bax-induced cell death 
in yeast and is rapidly upregulated during wounding and pathogen challenge. Plant $J$ 2000, $21: 393-399$.

48. Ihara-Ohori $Y$, Nagano $M$, Muto $S$, Uchimiya $H$, Kawai-Yamada $M$ : Cell death suppressor Arabidopsis bax inhibitor-I is associated with calmodulin binding and ion homeostasis. Plant Physiol 2007, 143:650-660.

49. Nakashima T, Sekiguchi T, Kuraoka A, Fukushima K, Shibata Y, Komiyama $S$, Nishimoto T: Molecular cloning of a human cDNA encoding a novel protein, DADI, whose defect causes apoptotic cell death in hamster BHK2I cells. Mol Cel Biol 1993, 1 3:6367-6374.

50. Lam E, Kato N, Lawton M: Programmed cell death, mitochondria and the plant hypersensitive response. Nature $A-Z$ Index 200I, 4I I:848-853.

5I. Liu Y, Schiff M, Czymmek K, Tallóczy Z, Levine B, Dinesh-Kumar SP: Autophagy regulates programmed cell death during the plant innate immune response. Cell 2005, I $21: 567-577$.

52. Mitsuhara I, Malik KA, Miura M, Ohashi Y: Animal cell-death suppressors Bcl-x L and Ced-9 inhibit cell death in tobacco plants. Cur Biol 1999, 9:775-778.

53. Dickman MB, Park YK, Oltersdorf T, Li W, Clemente T, French R: Abrogation of disease development in plants expressing animal antiapoptotic genes. PNAS 200I, 98:6957-6962.

54. Deising H, Jungblut PR, Mendgen K: Differentiation-related proteins of the broad bean rustfungus Uromyces viciae-fabae, as revealed by high resolution two-dimensional polyacrylamide gel electrophoresis. Arch Microbiol 1991, 155:191-198.

55. Mendgen K, Hahn M, Deising H: Morphogenesis and mechanisms of penetration by plant pathogenic fungi. Annu Rev Phytopathol 1996, 34:367-386.

Publish with Bio Med Central and every scientist can read your work free of charge

"BioMed Central will be the most significant development for disseminating the results of biomedical research in our lifetime. "

Sir Paul Nurse, Cancer Research UK

Your research papers will be:

- available free of charge to the entire biomedical community

- peer reviewed and published immediately upon acceptance

- cited in PubMed and archived on PubMed Central

- yours - you keep the copyright
BioMedcentral 